\title{
Identifying visual targets amongst interfering distractors: Sorting out the roles of perceptual load, dilution, and attentional zoom
}

\author{
Kyle R. Cave ${ }^{1} \cdot$ Zhe Chen $^{2}$
}

Published online: 1 June 2016

(C) The Psychonomic Society, Inc. 2016

\begin{abstract}
Visual selection is imperfect; whenever a complex array of objects is processed, representations of multiple objects are likely to be active simultaneously. A full account of attentional processing must explain how these representations affect one another and how they interact to produce a response. Evidence on these interactions comes from measures of distractor interference and from dilution of distractor effects by other nontargets. Based on these data, different principles have been proposed to help understand target-distractor interactions, including accounts based on perceptual load and on dilution among nontargets. We review evidence from a number of experiments, including some using Yantis and Jonides's (Journal of Experimental Psychology: Human Perception and Performance, 10, 601-621, 1984, Journal of Experimental Psychology: Human Perception and Performance, 16, 121134,1990 ) methods for preventing abrupt onsets, which can disrupt spatial attention. The results underscore spatial constraints on the allocation of attention to include targets and exclude distractors. Selection is most effective when a single region can be selected that includes all possible target locations and excludes possible distractor locations. This region can be expanded or contracted as needed for the task, as suggested by C. W. Eriksen and St. James's (Perception \& Psychophysics, 40, 225-240, 1986) zoom lens model. This attentional zoom setting is probably affected by a number of
\end{abstract}

Kyle R. Cave

kcave@psych.umass.edu

1 Department of Psychological and Brain Sciences, University of Massachusetts, Tobin Hall, 135 Hicks Way, Amherst, MA 01003, USA

2 Department of Psychology, University of Canterbury, Christchurch, New Zealand factors, including the number of nontargets, the similarity among stimulus elements, the discriminability of the possible targets, and the discrimination difficulty of a concurrent task. A narrower attentional zoom setting that excludes a distractor will prevent interference from that distractor. Interference from a distractor will be diluted by nontargets, but only if they are within the attentional zoom region.

Keywords Cognitive and attentional control · Visual search . Attention

When a visual target stimulus at a known location must be identified, attentional control systems will facilitate processing of the target by activating visual neurons with receptive fields including the target and inhibiting those with receptive fields including distractors (Serences \& Yantis, 2006; see also Yantis 2000, 2008). However, visual selection is not absolute. Some distractors will also be attended to some extent along with the target, especially those that are near the target, which are likely to be within the receptive fields of some of the same neurons representing the target. C. W. Eriksen and Hoffman (1973) and B. A. Eriksen and Eriksen (1974) demonstrated that these nearby distractors are processed to the point that they interfere with the response to the target. Even when target and distractor locations are known precisely, a weak signal from the distractor can leak through the selective filter if it is relatively close to the target. As a result, multiple items are represented simultaneously, with the strength of each item varying according to its salience and its level of attentional activation or inhibition.

The processing of distractors can affect the response to the target in multiple ways. The distractors might compete with the target for processing resources, and they might activate competing responses. Also, activation in distractor representations 
might increase or decrease activation in other representations, depending on the similarity of the represented items. To understand visual recognition, we must be able to explain the complex interactions among these different visual objects.

When there is one target, strengthened by attentional selection, and one distractor, the interaction is fairly straightforward. However, when more distractors are added to the display, the interactions become more complex. These additional distractors interfere with the processing of the target, of course, but they also interfere with one another, so that one distractor can "dilute" the interference from another distractor (Kahneman \& Chajczyk, 1983; Tsal \& Benoni, 2010; Wilson, Muroi, \& MacLeod, 2011). This dilution among distractors has come to play an important role in understanding these interactions, as described below.

Many of the experiments exploring the interactions between targets and distractors have been done with simple letter and number stimuli. Changing the configurations of a relatively small number of easily identifiable features produces a number of different stimulus identities. The high familiarity of these stimuli makes it easy for subjects to learn and remember which are targets and which are distractors for a particular task, and the subjects have had lots of practice in distinguishing them from one another.

A number of different experimental paradigms have been used to measure the interactions among targets and different classes of nontargets (e.g., task relevant vs. irrelevant and/or response relevant vs. irrelevant), resulting in a complex set of results to be explained. A diverse set of different theoretical accounts has been offered to interpret these data, and they will be examined and compared below. We will start with Lavie's (1995, 2005; Lavie \& Tsal, 1994) perceptual load theory (PLT) and the four different types of evidence offered to support it. Then, we will review experimental evidence that has led to a series of alternative accounts, including the dilution accounts by Tsal and Benoni (2010) and Wilson et al. (2011), and a detailed model recently presented by Neokleous, Shimi, and Avraamides (2016). One important factor in these experiments is the broadening of attention that comes from sudden onsets; once these effects are controlled with methods from Yantis and Jonides (1984, 1990), the resulting data fit suggests that performance on these tasks is shaped by an attentional zoom mechanism that adjusts the size of the attended region according to the level of interference between different stimulus elements.

\section{Perceptual load}

Finding order among the stimulus interactions In an attempt to impose order over the apparent free-for-all of competition and inhibition among different visual objects, Lavie (1995, 2005; Lavie \& Tsal, 1994) proposed PLT. This framework offered an explanation of these interactions that was plausible, straightforward, and easy to understand. Like many other theories, it assumes that there is a fixed amount of processing resources to be distributed across all the visual stimuli that are present. Unlike many other theories, though, PLT asserts that all of the capacity will be used. The stimuli that are relevant to the current task will receive the resources required for their processing first. Any and all additional resources will then be allocated to irrelevant stimuli. This seems like a prudent use of resources that ensures that current processing goals will be met and maximizes the amount of information taken in from the environment at any given moment.

If those irrelevant stimuli receive enough processing, they can interfere with the response to the target. Thus, PLT makes the surprising and testable claim that increasing the resources necessary for the current task will deny resources to irrelevant stimuli and will thus limit distractor interference. This claim is often tested by presenting a target along with a very salient distractor. The distractor can be either congruent or incongruent with the target response, and the effects of a congruent and an incongruent distractor are compared to determine the amount of distractor interference. When the task is changed in a way that increases the resources it requires (its perceptual load, or PL), the interference from the salient distractor should decrease.

The experiments testing the relationship between PL and distractor interference generally fall into four categories, which are described more fully in Chen and Cave (2016). In many of these experiments, there are other stimuli in the display besides the target and the salient distractor, and they are usually referred to as nontargets to distinguish them from the salient distractor. In the first group of studies (see Fig. 1A), PL is increased by adding additional relevant nontargets to the display so that a search is necessary to find the target among these nontargets, especially in the high-load condition (Kumada \& Humhreys, 2002; Lavie, 1995, Experiment 1; Muggleton, Lamb, Walsh, \& Lavie, 2008). In the second group (see Fig. 1B), PL is increased by increasing the similarity between the target and the nontargets and among the nontargets (Carmel, Saker, Rees, \& Lavie, 2007; Lavie \& Cox, 1997). In the third group (see Fig. 1C), PL is increased by making it more difficult to discriminate among the possible targets (Bahrami, Carmel, Walsh, Rees, \& Lavie, 2008; Brand-D'Abrescia \& Lavie, 2007; Cartwright-Finch \& Lavie, 2007; Couperus, 2001; Handy \& Mangun, 2000; Taya, Adams, Graf, \& Lavie, 2009). In the fourth group (see Fig. 1D), which will be considered in more detail later, the target discrimination task is combined with a go/no-go task, and the difficulty of the go/no-go task is manipulated (Lavie, 1995, Experiments 2A and 2B). In all these four groups of studies, distractor interference is larger when PL is low compared with when PL is high, a result consistent with PLT. 


\section{Low Load High Load}

A Small set-size
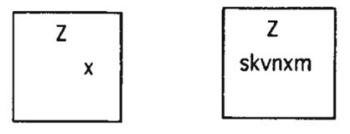

Large set-size

B Efficient
search

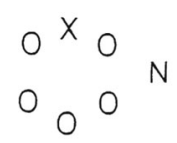
$\begin{array}{lll}M^{K} Z & & \text { Inefficient } \\ H_{W} & & \end{array}$

C

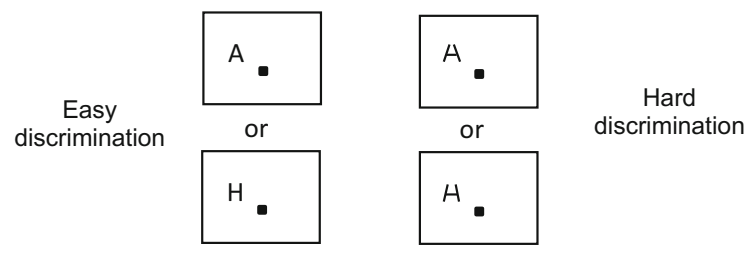

D

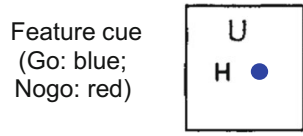

distractor, a very large and salient singleton completely different from the stimuli in the letter task (e.g., a cartoon SpiderMan) would appear outside the task-relevant region. In these irrelevant singleton trials, the effect of the singleton on RT depended on the duration of the letter display. When the stimulus display remained on the screen until response, the cost of the singleton in RT was comparable between the low-and high-PL conditions. In contrast, when the letter display was shown for only $100 \mathrm{~ms}$, the presence of the singleton did not increase RT in the high-load condition, and this pattern of result was found regardless of whether the singleton was shown for $100 \mathrm{~ms}$ or remained on the screen until response. Forster and Lavie concluded that PL could determine whether a completely irrelevant distractor was attended or not; furthermore, the degree of distractor processing was influenced by the presentation duration of the task-relevant stimuli instead of the task-irrelevant distractor - a result consistent with the finding of Roper and Vecera (2013).

Exactly why the effect of high PL on an irrelevant singleton should vary as a function of stimulus presentation duration in Forster and Lavie (2008b) is unclear from PLT. However, it is plausible that the short presentation duration of the target display induced the participants to evoke stronger top-down control in their attentional setting, especially in the high-load condition block. The stronger top-down control could be in the form of greater attentional inhibition surrounding the taskrelevant array and/or a smaller attentional zoom that excluded the distractor, resulting in the absence of distractor interference.

Another factor that can affect attention to these completely irrelevant distractors is the presence or absence of distractors with shapes that make them relevant to the response. When a response-relevant distractor never appeared in the target display, the effect of PL was different in different studies. Whereas PL did not appear to affect the magnitude of the interference effect caused by the task-irrelevant singleton in some studies, regardless of whether the display duration of the relevant stimuli was long (Biggs, Kreager, Gibson, Villano, \& Crowell, 2012) or short (Forster \& Lavie, 2008a, Experiment $1)$, an increase in PL reduced distractor processing in other experiments (Forster \& Lavie, 2008a, Experiments 3 and 4).

It is unclear to what degree the observed pattern of results in the aforementioned experiments is attributable to variation in PL, but a high PL appears to be able to eliminate the interference of a highly salient, task-irrelevant singleton when a target display never includes a response-relevant distractor. In Forster and Lavie (2008b), Experiment 4, none of the target displays had a response-relevant distractor, and perceptual load was varied within a block. The task-irrelevant singleton increased RT in the low- but not the high-load condition.

Do PL effects extend beyond visual processing? There have been inconsistent results in the literature regarding the 
relationship between the degree of distractor processing in selective attention tasks and everyday cognitive failures in general, as measured by Cognitive Failures Questionnaire (Broadbent, Cooper, FitzGerald, \& Parks, 1982). Using a Stroop task, Martin (1983) found no association between the two phenomena. In contrast, using a typical perceptual-load task with low- and high-load trials in separate blocks, Forster and Lavie (2007) found a positive relationship between cognitive failures and distractor processing in the low-load task but not in the high-load task. Forster and Lavie interpreted their results as indicating that the level of perceptual load in a task can predict individual differences in distractibility. However, as discussed below, it is possible that the pattern of data observed in Foster and Lavie was caused, at least in part, by their blocked design, which would have allowed differences in attentional zoom across the different load conditions.

Forster and Lavie (2009) took things a step further. Their participants reported more unrelated thoughts in a low-load condition than in a high-load condition, leading Forster and Lavie to conclude that when engaged in a high-load task, participants work to exclude more unrelated thoughts. The probe method used to measure unrelated thoughts in these experiments was less than ideal, but it nonetheless produced a significant difference between low-load and high-load conditions that needs to be explained. It suggests that the attentional filtering that occurs in difficult visual tasks extends beyond the visual system to higher level cognition.

This effect may arise in part because of issues of attentional control. Perhaps maintaining a narrow attentional zoom requires more high-level effort, making it harder to entertain unrelated thoughts at the same time. Another important factor to consider is that even when attentional zoom is at a very narrow setting, it is probably not completely excluding visual information from outside the attentional focus. Some information will still be getting through, which means that there will be more high-level work to do in high-load trials than in lowload trials in sorting out the information that makes it through attentional selection. This extra high-level processing may make it more difficult for unrelated thoughts to be processed.

Block order can affect top-down attentional allocation An important feature in the vast majority of PL studies is the use of a block design, in which the high- and low-PL conditions were run in separate blocks (e.g., Foster \& Lavie, 2007, 2008a, b; Lavie, 1995; Lavie \& Cox, 1997). Indeed, when the trials in the different load conditions were intermixed within a block, no effect of PL was found (Benoni, Zivony, \& Tsal 2014; Murray \& Jones, 2002; Theeuwes, Kramer, \& Belopolsky, 2004; see also Biggs \& Gibson, 2010, for evidence of distractor processing as a function of intertrial contingency rather than the level of perceptual load). This pattern suggests that distractor processing may not depend so much on the perceptual load of the current stimulus but rather on the participants' expectations about the processing demands of the task based on their experience with previous trials.

The block order effect observed in these experiments is consistent with previous research showing that distractor processing is modulated by both selection history and topdown search strategies (Chen \& Cave, 2015; Leber \& Egeth, 2006a, b; Zehetleitner, Goschy, \& Müller, 2012; see also Awh, Belopolsky, \& Theeuwes, 2012, for a review on the role of search history in the allocation of attention). The effects of selection history were demonstrated by Leber and Egeth and by Zehetleitner et al., who showed that when participants were induced to use one of two search modes in completing a task in the training phase they continued to use the same search mode in the subsequent test phase, even though the task could be performed with a different search mode. Chen and Cave also reported that in singleton-search tasks, the participants who began the experiment with neutral cues were more likely to ignore informative cues later in the experiment.

The separation of trial types into separate blocks may help to explain why PL effects appear more readily with short display times, as described above. The use of the block design may also have contributed to some of the inconsistencies in the experiments on cognitive failures. Block order has also been shown to affect the results of experiments that use go/no-go cues to manipulate attentional demands. These results will be discussed below. The effects of expectation that are illustrated in these manipulations of block order are not explained by PLT. However, they are consistent with an alternative account that assumes that the expectation of high interference leads to a narrowing of attentional zoom.

Problems with relevance, stimulus timing, and other factors PLT gains plausibility because it is consistent with converging evidence from multiple different experimental procedures. In some of those studies, the exact effect of PL appeared to depend on a number of factors, including participants' age, knowledge about the stimuli, and affective valence evoked by the distractor. Huang-Pollock, Carr, and Nigg (2002) showed that compared to young adults, children showed no distractor interference at lower perceptual loads, presumably because children have smaller capacity than young adults due to immaturity of their brains. Ro, Friggel, and Lavie (2009) compared the performance between musicians and nonmusicians in a selective attention task that required the participants to search for a name of a musical instrument and to determine whether it was a string or a wind instrument. Interference effect was larger in musicians than in nonmusicians. Practice in music apparently made the search task easy, resulting in 
greater distractor processing due to reduced processing load needed to perform the search task. Biggs et al. (2012) reported that although a distractor evoking mild negative affect did not capture attention in high PL trials, a distractor evoking a strong negative affect did. Taken together, these results suggest that although PL is not the determining factor in attentional selection, as suggested by Lavie's (1995) original PLT, it is one of multiple factors that can affect distractor processing.

As PLT is tested in different ways, other results emerge that do not fit so well with the theory, and it begins to look like its simple principles do not fully capture the complex interactions among targets and nontargets in complex stimulus arrays. For example, Kyllingsbæk, Sy, and Giesbrecht (2011) tested the central claim of PLT that the current task receives all the resources it needs. They showed that performance on a multipleletter report task decreases as more irrelevant distractors are added to the display, especially when they have the same color as the target. This result demonstrates that, contrary to PLT's claims, resources to the stimuli relevant to the task vary depending on irrelevant parts of the display.

A variety of other studies raise other problems for PLT. A valid precue indicating the location of the target eliminates the PL effect (Chen \& Cave, 2013, 2014; Johnson, McGrath, \& McNeil, 2002; Miller, 1991; Paquet \& Craig, 1997), as does confining the relevant and irrelevant information to the same object or spatial region (Chen, 2003; Taya et al., 2009), or prolonging the duration of the target display until response (Roper, Cosman, Mordkoff, \& Vecera, 2011; Roper \& Vecera, 2013). A distractor or nontargets that are in the same hemisphere as the target will reduce distractor processing compared to a distractor or nontargets in different hemispheres (Torralbo \& Beck, 2008; Wei, Kang, \& Zhou, 2013). An infrequently presented onset distractor leads to significant distraction processing while a frequently presented one does not (Cosman \& Vecera, 2010), and both perceptual grouping and the salience of a target relative to the distractor and/or the other stimuli in a display affect the degree of distractor processing (Baylis \& Driver, 1992; Biggs \& Gibson, 2013; Biggs, Kreager, \& Davoli, 2015; Cosman \& Vecera, 2012; Eltiti, Wallas, \& Fox, 2005; Yeh \& Lin, 2013; Yeshurun \& Marciano, 2013). These and other empirical findings (Benoni \& Tsal, 2010; Biggs \& Gibson, 2010; Chen \& Chan, 2007; Tsal \& Benoni, 2010; Wilson et al., 2011; Yeh, Lee, Chen, \& Chen, 2014 ), together with the conceptual and methodological issues raised by a number of researchers (see Benoni \& Tsal, 2013; Giesbrecht, Sy, Bundesen, \& Kyllingsbæk, 2014; and Murphy, Groeger, \& Greene, 2016, for reviews), indicate that the simple principles of PLT do not fully capture the complex interactions among targets and nontargets in complex stimulus arrays. In this review we will focus primarily on behavioral studies that do not involve a secondary task. (For dual-task studies that involve working memory load, see de Fockert, 2013, for a review.)

\section{Dilution}

Dilution accounts The pure form of PLT assumes that the current relevant task requires a fixed amount of resources, and that its processing will not suffer if the remaining resources are applied to irrelevant stimuli. The visual system may not achieve such clear separation between the processing of the relevant and the irrelevant stimuli. It might be possible to accommodate the experimental results described above by creating a modified version of PLT that has flaws in the mechanism for protecting the processing resources allocated to the relevant stimuli, and that takes into account the encoding demand of the task relevant stimuli. However, another set of experiments tests the distinction between relevant and nonrelevant stimuli in a different and very fundamental way. PLT claims that the interference from a salient distractor can be reduced by adding additional relevant nontargets to the display. The additional nontargets will have to be processed up to the point that they can be eliminated as targets, and thus they will add to the perceptual load. Rather than adding to the number of relevant items in the display, Tsal and Benoni (2010; Benoni \& Tsal, 2010) added irrelevant stimuli and found that they also reduced the interference from the salient distractor. Wilson et al. (2011) found similar results using a stimulus arrangement that made the distractor particularly salient by positioning it at fixation, while the target and other nontargets were positioned on an imaginary circle around fixation. Based on these findings, both groups proposed that the decrease in distractor interference is not from resources that are allocated by stimulus relevance, but is simply from dilution among the nontargets.

Results consistent with the dilution account have been found in a number of other studies, both in healthy young adults (Chen \& Cave, 2013, 2014; Yeh \& Lin, 2013) and in patients with extinction and/or neglect (Mevorach, Tsal, \& Humphreys, 2014). In the latter study, Mevorach et al. manipulated both the number of nontargets and their location. When multiple nontargets were presented, they were aligned vertically along the vertical meridian, in the contralesional side of space, or in the ipsilesional side of space. Importantly, compared to the no nontargets condition, adding nontargets in the contralesional side of space had the same diluting effect as adding nontargets along the vertical meridian, even though stimuli presented in the contralesional space should receive reduced attention due to neglect, and this in turn should have led to increased distractor processing according to PLT. Together, these studies show that the perceptual load effect reported in many previous studies, 
especially those that manipulated perceptual load by varying the number of task relevant nontargets in the display (e.g., Lavie, 1995, Experiment 1; Muggleton et al., 2008), may in fact result from dilution.

Exactly where dilution occurs in the stream of visual processing was not clear from the studies by Tsal and Benoni (2010; Benoni \& Tsal, 2010) or Wilson et al. (2011). Both preattentional and postattentional accounts of dilution were suggested. Tsal and Benoni left open the possibility that dilution occurs very early in visual processing, with features from one object interfering with those in other objects before attentional selection. Wilson et al. proposed a more specific account, which was built upon Neisser's (1967) and Hoffman's (1979) two-stage account of visual processing. They suggested that dilution occurs in the second stage, after a single display item has been selected for thorough processing. The nonselected items compete for processing resources not needed for the selected item, and this competition allows nontargets to dilute the effects of the salient distractor.

Processing obscured by abrupt onsets In interpreting these experimental results, it is important to consider some principles that have emerged from work in other types of visual attention research. One of those principles is the propensity for visual attention to be allocated to stimuli that suddenly appear out of nowhere. Yantis and Jonides $(1984,1990)$ demonstrated this attentional capture by abrupt visual onsets, and it is clear that this capture could be affecting processing in many of the experiments described above, because in many of them a stimulus array appears suddenly, superimposed on a background that was previously fairly empty. This array of abrupt onsets has the potential to broaden the allocation of spatial attention to cause the entire array to be processed more fully than it otherwise would be. This broadening of attention could have an effect on just about all of the results described above, because demonstrations of both perceptual load effects and dilution effects have often used stimulus arrays that were presented as sudden onsets.

Luckily, Yantis and Jonides $(1984,1990)$ provided an experimental method for controlling attentional capture by abrupt onsets. Rather than starting the trial with an empty display that was suddenly replaced by an array of characters, they started each trial with an array of figure-eight stimuli made from a collection of straight lines. Each of these block figures was positioned at a location that would have a character in the stimulus array. When the time came to display the characters of that array, they were created by removing a few lines in each of the figure eights to create the character for that location. Thus, rather than each character appearing as an abrupt onset, it appeared as the offset of a few contours that were among other contours that remained static throughout the trial.
Eltiti et al. (2005) realized that in many PL experiments with onset stimuli, a distractor was more likely to be attended and interfere with the response in the low PL condition than in the high PL condition, because the distractor was more salient in the low-load condition, in which the display contained few stimuli or homogenous nontargets dissimilar to the distractor, than in the high-load condition, in which the distractor was very similar to both the target and nontargets. They showed that when the distractor was instead created by removing segments from a Yantis and Jonides (1984) style figure eight, it no longer interfered with the response. Because this offset distractor eliminated interference even in a low-PL stimulus array, Eltiti et al. offered their own salience account of distractor interference as an alternative to PLT. Support for the salience account has also been found in Benoni and Tsal (2012); Yeshurun and Marciano (2013); and Roper, Cosman, and Vecera (2013). Roper et al. showed that the degree of distractor processing was determined primarily by targetdistractor similarity rather than distractor-distractor similarity, with the latter contributing to distractor processing only when search was inefficient. In addition, the degree of distractor processing correlated negatively with search inefficiency: the larger the search slope, the smaller the response congruency effect. These results prompted Roper et al. to propose that PL is a continuous variable better defined operationally by search efficiency rather than task difficulty. (See also Biggs et al., 2015 , for evidence on the interaction between visual search and PL.)

Chen and Cave (2013) went even further in controlling salience. They created the entire stimulus array by removing segments from figure eights to avoid all sudden onsets. They combined Yantis and Jonides' $(1984,1990)$ method for presenting letter stimuli without sudden onsets with Wilson et al.'s (2011) procedure for measuring dilution. Their stimulus arrays, like those from Wilson et al., consisted of a distractor letter at fixation and six additional letters equally spaced in a ring around fixation. The target was one of two letters $(\mathrm{H}$ or $\mathrm{S})$ and appeared at one of the six locations around fixation. The central distractor was also either an $\mathrm{H}$ or an $\mathrm{S}$, and even though subjects knew they could ignore it, it nonetheless interfered with target processing as would be expected from Eriksen's experiments: Responses were faster when the central distractor matched the target and slower when it did not match.

Dilution occurs only within the attended region The amount of distractor interference in Chen and Cave's (2013) experiments varied with the number of nontargets that appeared on the ring with the targets, just as it did for Wilson et al. (2011). However, as described below, interference also varied according to whether the nontargets were task relevant or irrelevant, a result inconsistent with the prediction of the dilution accounts. 
In one experiment, Chen and Cave (2013, Experiment 2) used a spatial cuing paradigm to cue the potential target locations (see Fig. 2 for examples of the stimuli). In half the trials, all six locations on the target ring were cued. These trials produced the standard dilution effect. In one condition (the two-letter-six-cue condition), the target could appear with equal probability at any of the six locations on the ring. When segments were removed from one figure eight to produce the target letter $(\mathrm{H}$ or $\mathrm{S})$, segments were also removed from the central figure eight to produce the salient distractor $(\mathrm{H}$ or $\mathrm{S})$ and from one other figure eight on the ring on the side opposite the target to produce a nontarget letter $(\mathrm{P}, \mathrm{E}, \mathrm{F}, \mathrm{L}$, or $\mathrm{U})$. The other four items on the ring remained figure eights throughout the trial. In this condition, there was effectively only one nontarget letter to dilute the effect of the central distractor, and the interference from the distractor was substantial. In another condition (the six-letter-sixcue condition), all the figure eights on the ring were converted to letters. With five nontarget letters surrounding the central distractor rather than just one, the representation of the central distractor was apparently weakened, because the distractor interference decreased. This pattern replicated Wilson et al.'s (2011) dilution: The extra nontargets diluted the effect of the central distractor on the response. This result demonstrated dilution in a context in which attention is not broadened by abrupt onsets.

Interestingly, dilution disappeared in the other half of the trials, in which just two of the six locations on the target ring were cued. The two cued locations were always on opposite sides of the ring so that the central distractor was right between them, making it difficult to ignore. The cue was always valid: The target always appeared at one of the two cued locations so that subjects could adjust their spatial attention to exclude the four uncued locations on the ring. As in the six-cue trials, the nontargets consisted of either two letters or six letters.

The cues made four of the six ring locations irrelevant to the task, and thus PLT predicts that it should lower the perceptual load and raise the interference from the central distractor. Instead, distractor interference was lower in the two-cue trials than in the six-cue trials. The results also demonstrate something important about the nature of dilution, because dilution disappears in the two-cue condition. When the four nontarget letters can be excluded by spatial attention, they no longer dilute the effect of the central distractor: Interference from the central distractor was no less with six letters on the ring than it was with two letters on the ring. This result suggests that dilution arises only from letters at cued locations. Based on this result, Chen and Cave (2013) concluded that dilution is confined to the region selected by attentional zoom: The two-cue condition allowed participants to narrow the attended region to exclude the four noncued nontargets, and this prevented them from diluting the effects of the central distractor.

Evidence consistent with an attentional zoom account has also been demonstrated in a number of other studies. Cosman and Vecera (2009, Experiment 2; 2010, Experiments 1 and 3) found no congruency effect in either

Cue Display (4 frames, each $500 \mathrm{~ms}$ )

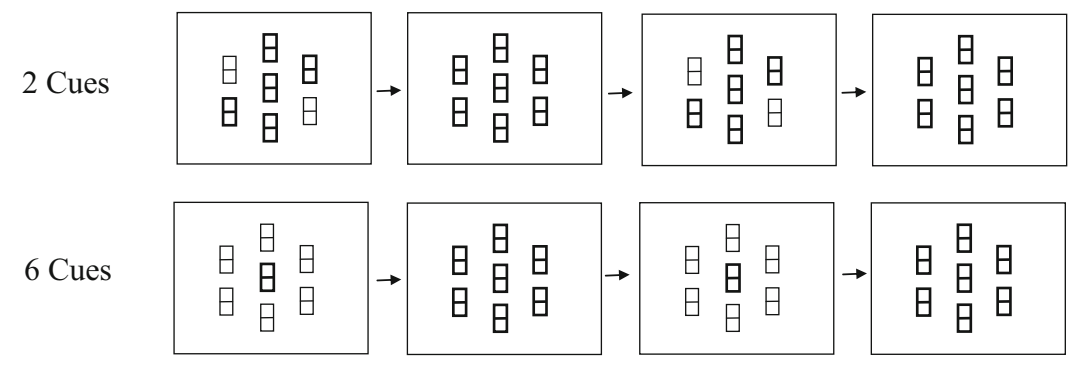

Target Display (200 ms)

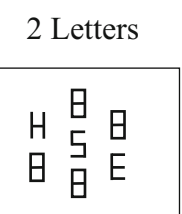

Fig. 2 Examples of cue displays and target displays from Chen and Cave (2013) Experiment 2. Cue locations were indicated by two successive luminance decrements at the beginning of the trial. The locations of the target, which was either an $\mathrm{H}$ or an $\mathrm{S}$, were indicated by two or six figure-
6 Letters

$$
\begin{array}{lll}
H & P & \\
L & H & E
\end{array}
$$

eight placeholders, increasing in luminance. The target display consisted of two letters or six letters, excluding the critical distractor, which always appeared at the center. The appearance of the target display is signaled by luminance decrement 
the low- or the high-load condition when the distractor, together with the other stimuli in the display, was a luminance offset. Dilution occurred only when the distractor was a luminance onset or a motion onset while the other stimuli were offsets. Gaspelin, Ruthruff, and Jung (2014) cued either the target or the distractor location in displays with varying numbers of nontargets. According to PLT, cuing the target location should make nontargets irrelevant and reduce PL, and thus PLT predicts that the distractor will be attended more and will therefore interfere with the response more. Instead, however, the target cue eliminated most of the interference; a result consistent with Johnson et al. (2002) and Paquet and Lortie (1990). Cuing the distractor, on the other hand, led to more distractor interference. Based on these results, Gaspelin et al. offered an account of the results from PLT experiments (slippage theory) based on an early-selection mechanism that occasionally misallocated attention to the distractor. This "slippage" was more likely when there were few other nontargets competing for attention.

In the above experiments, the spatial cues were always $100 \%$ valid: The target could never appear at an uncued location. This procedure makes it more likely that the attentional system will zoom in on the cued locations and will exclude the uncued locations as fully as possible. In order to understand how spatial attention shapes dilution, it is also informative to measure dilution in a procedure that includes some invalid trials so that the target sometimes appears at an uncued location. In Chen and Cave (2014), Experiment 1, the target appeared at one of the four uncued locations on $40 \%$ of the trials $(60 \%$ validity). Dilution was absent on the valid trials, in which the ring locations that were attended included the target and just one nontarget. On the invalid trials, the two ring locations within the attentional zoom included two nontarget letters, which were processed until it was determined that neither one could be a target. Thus, these letters were processed long enough and fully enough that their representations could interfere with the central distractor, as shown by a strong dilution effect.

Together, these experiments show that dilution is an interaction among items that are within the attentional zoom. When the attentional zoom can be narrowed to exclude nontarget letters, those letters do not contribute to dilution.

Dilution at a level above simple features The fact that nontarget letters produce more dilution than nontarget figure eights in Chen and Cave $(2013,2014)$ suggests that dilution arises because representations of the nontarget letters are competing with the representation of the central distractor. When the nontargets do not activate letter representations, they do not produce the same level of dilution. The role of letter representations in dilution was further tested in Chen and Cave (2013), Experiment 3. In that experiment, the nontarget letters that appeared on four locations on the ring were replaced with inverted letters. The inverted letters should match the upright letters in basic features but should not activate the letter representations. This change to inverted letters eliminated dilution, showing that in these displays with target and distractor letters, the central distractor is diluted by activation from nontarget letter representations. A related result was reported in Experiment 4 of Thoma and Lavie (2013). In that experiment, the target and distractor were both faces. In different experimental conditions, the nontargets were multiple faces (the high-load condition), multiple scrambled faces (the dilution condition), or a single face (the low-load condition). Compared to the low-load condition, dilution was found in the high-load condition but not in the dilution condition, even though the nontarget stimuli were similar at the feature level in both the high and dilution conditions.

In these experiments, the dilution that occurs probably reflects competition among the representations that participants choose to use for the task. Because the tasks require distinguishing among letters or faces, competition will occur among these higher level representations. If a task involved unfamiliar shapes or scrambled images, then perhaps dilution would occur among representations of more basic features. For the tasks used in these experiments, the interference and dilution among the different stimuli probably cannot be explained solely as effects of stimulus salience, or solely as interactions among basic features that are identified early in visual processing.

Color segmentation can also protect against dilution The experiments reviewed above show that a salient distractor that is attended is insulated against competition from nontargets outside the attended region. Another set of results demonstrates that a distractor representation can be protected from interference by color segmentation. This protection by color can be seen in Chen and Cave's (2014) Experiment 4A and $4 \mathrm{~B}$, which used the same arrangement of stimuli and the same $60 \%$ valid spatial cues as in Chen and Cave's (2014) Experiment 1 . Thus, on invalid trials, there were two nontargets at the cued locations that could potentially dilute the effects of the central distractor. In these experiments, however, the distractor was a color singleton while the target and the nontargets shared a different color. This color segmentation prevented dilution. Within the attentional zoom region, items were apparently grouped by shared features, and interference across groups was limited. The central distractor may also have benefited from an extra attentional boost because it had a unique color within the display. 
Similar results were reported by other studies (Biggs \& Gibson, 2014; Yeh \& Lin, 2013; but see Gaspelin, Ruthruff, Jung, Cosman, \& Vecera, 2012). ${ }^{1}$ In Biggs and Gibson, participants saw displays that consisted of two concentric rings. The target letter could only occur in the inner ring while the critical distractor letter was in the outer ring. The nontargets in the outer ring varied depending on the experimental condition. They were dashes in the low-load condition, letters having the same color as that of the distractor in the high-load condition, or letters having a different color from that of the distractor (i.e., the distractor was a color singleton) in the high-dilution condition. Compared with the low-load condition, dilution was found in the high-load condition but not in the highdilution condition. No dilution was found in the singleton distractor condition in Yeh and Lin's study, either. Taken together, these results show that when a distractor is a color singleton, the extra attention it attracts protects its representation from the diluting effect of the nontargets.

The distractor in the experiments just described was a color singleton. Because color singletons capture attention, protection from dilution could arise in these experiments purely in a stimulus-driven/bottom-up way. Other results, however, show that dilution can also be prevented by advance knowledge of the target. In Chen and Cave (2013), Experiment 4, the target shared its color with only one nontarget in the display (the one on the opposite side of the ring). The other four nontargets were of a different color, which they shared with the central distractor. If participants knew the target color in advance, they could quickly and efficiently select the two locations with the target color (and perhaps also the central distractor between them). This color-driven selection prevented dilution from the other four letters on the ring.

However, dilution was reported by Benoni and Tsal (2010) and Tsal and Benoni (2010) even when participants knew the color of the target in advance. In their experiments, the target and distractor were both letters. The nontargets could be completely absent or they could be an array of heterogeneous letters similar to the target, an array of homogenous letters dissimilar to the target, or

\footnotetext{
${ }^{1}$ Gaspelin et al. (2012) manipulated the salience of the distractor (which could be either a color singleton or not) in addition to the perceptual load required of the task (nontargets homogenous vs. heterogeneous). Unlike other studies (e.g., Chen \& Cave, 2013, 2014; Yeh \& Lin, 2013), there was no evidence that the salience of the distractor affected the degree of distractor processing. Although it is unclear what caused the different results in these studies, it should be noted that the size of the stimulus in Gaspelin et al. was very large. The task relevant stimulus subtended $2.1^{\circ} \times 2.5^{\circ}$ each, and the distractor $3.4^{\circ} \times 3.8^{\circ}$. The target ring was $12.1^{\circ}$ in diameter, and the distractor was a further $1.9^{\circ}$ away from the ring. These stimulus features may have rendered the distractor relatively easy to inhibit, resulting in the absence of the distractor singleton effect. This may also explain why distractor interference was reduced, rather than increased, in the low-load singleton condition compared with the lowload nonsingleton condition, suggesting that stronger inhibition was applied to the distractor when it was a singleton.
}

homogenous symbols such as dots or dashes. The target and nontargets were either the same color or different colors, and the color of the target was known in advance or unpredictable. The dilution of distractor interference appeared to depend on the heterogeneity of the nontargets instead of the foreknowledge of the target color. Whereas significant congruency effects were found when the nontargets were homogenous and dissimilar to the target, no congruency effect was found when they were heterogeneous and similar to the target, and this pattern of data did not change when the color of the target was known in advance.

Tsal and Benoni (2010; Benoni \& Tsal, 2010) concluded that the nontargets were diluting the influence of the salient distractor. These results might be evidence that nontargets can sometimes dilute a salient distractor even though their color makes it possible to attentionally exclude them. Before reaching that conclusion, however, there are two factors to consider that might explain these results. First, in these experiments the stimuli were presented as sudden onsets, and as described above, this could trigger a spread of attention to encompass a wide region across the display. Once the attentional zoom is expanded, it would allow the nontargets to be more fully processed and to dilute the salient distractor. Second, the trials in different experimental conditions were presented in separate blocks. As the target displays differed in multiple ways across the different conditions in these studies, the participants could adopt different attentional control settings in separate blocks, and, as discussed both above and below, such differences in attentional control setting can affect the degree of distractor processing.

\section{Attentional zoom}

These results show that attentional settings affect the interference between stimulus items in a complex visual array. When attention is zoomed in to a small part of the visual field, nontargets outside of this selected region are less able to dilute the interference from a salient distractor. Given that attentional zoom provides a plausible account of dilution experiments when the effects of sudden onsets and blocked conditions are controlled, the question arises as to whether the results of the original experiments demonstrating PL can also be attributed to attentional zoom. In general, the four different types of manipulations listed above that have been used to increase perceptual load are the sorts of manipulations that would be expected to increase interference between target and nontargets, and thus these manipulations would probably make a narrow attentional zoom more advantageous because it would limit interference. 
As described above, the first method for increasing perceptual load is by adding relevant nontargets to the display. As the display includes more items that can interfere with the target, there is more incentive to narrow attentional zoom to prevent that interference. The second method is to increase similarity between the target and the nontargets. Again, this manipulation will increase the target/nontarget confusability and thus produce additional incentive for narrow attentional zoom. The third method is to increase similarity across the possible targets, which might make it necessary to collect and analyze more information about each item to determine if it is the target. This extra processing demand may make it necessary to limit the total amount of information taken in at any one moment, which could be accomplished by narrowing attentional zoom.

The previous section postponed consideration of the fourth method, so it will be examined here in some detail.

\section{Manipulating attentional zoom with a cue at fixation In the} fourth method that has been used to demonstrate PL effects, the target identification task is combined with a go/no-go task that requires the identification of a single cue stimulus at fixation. A response is made only for one type of cue, and the response is withheld for the other type. In Lavie (1995), Experiment 2, there were "low-load" and "high-load" conditions. In the low-load condition, the "go" cue was distinguished from the "no-go" cue simply by color. In the high-load condition, on the other hand, information about the cue's color had to be combined with information about its shape. The choice of these two types of cues was based on Treisman and Gelade's (1980) distinction between feature tasks that could be performed preattentively and conjunction tasks that required focused attention.

The extra difficulty in the high-load condition might have arisen from the need to conjoin information from two different feature dimensions, as Lavie originally assumed, or the shape discrimination in the high-load condition might have been more difficult than the color discrimination in the low-load condition. However, it is clear that the more difficult cue task affected processing of a salient distractor because interference from that distractor was reduced in the high-load condition relative to the low-load condition. Thus, the results from this fourth method fit nicely with those from the other three methods: high PL once again led to less distractor interference.

None of the alternative accounts to PLT described so far have addressed this demonstration of PL effects based on easy versus difficult go/no-go cues. However, these results do seem to fit within the attentional zoom account. In fact, although there is not direct evidence that the other three methods produce changes in attentional zoom, there is evidence from event-related potential (ERP) and steady state visually evoked potential (SSVEP) measurements that the low-load and highload cues used by Lavie (1995) manipulate attentional zoom. Handy, Soltani, and Mangun (2001) used low-load cues

defined by color and high-load cues defined by a conjunction of color and orientation. When a target appeared at fixation, the the first positive peak after stimulus onset (P1) that it generated did not vary across the low-load and high-load conditions. However, a probe that appeared more peripherally generated a stronger P1 in the low-load condition than in the high-load condition, suggesting that attention was more narrowly focused with the high-load cue.

A similar cue task also produced measurable changes in attentional zoom in a study by Parks, Beck, and Kramer (2013). In this case, attentional zoom was measured with a flickering ring encircling fixation at a distance of $2^{\circ}, 6^{\circ}$, or $11^{\circ}$ of visual angle. The SSVEP generated by the ring at $2^{\circ}$ was stronger for a cue requiring a luminance discrimination than for a cue requiring a combination of luminance and orientation information, suggesting that the region $2^{\circ}$ around fixation was inhibited more during the conjunction task. Once again, a cue task that would be classified as "high load" by PLT led to more narrowly focused attention.

This link between go/no-go cues and attentional zoom means that the changes in distractor interference found by Lavie (1995) might be due to differences in attentional zoom settings. This possibility was tested in a series of experiments by Chen and Cave (2016), in which a small target letter had to be identified in the presence of a larger distractor letter. Trials began with a go/no-go cue. The easier condition required a discrimination of the cue color, while the more difficult condition required that color and shape information be combined. In Chen and Cave's Experiment 2, the interference from the distractor letter was greater with the easy cue task, as predicted by PLT. However, in that condition, the target letter was always at one of two possible locations next to fixation, and the distractor was positioned near the target but farther away from fixation (see Fig. 3B). This stimulus arrangement made it

\section{A Stimuli from Experiment 1.}

\section{Congruent}

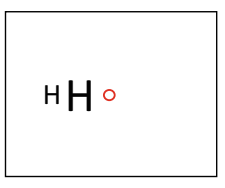

\section{B Stimuli from Experiment 2.}

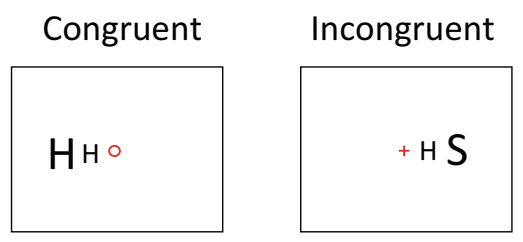

Fig. 3 Examples of stimulus displays from Chen and Cave (2016). The target was the smaller of the two letters. (A) Experiment 1. (B) Experiment 2 
possible to adopt a narrow attentional zoom setting that selected both possible target locations and excluded both possible distractor locations. Thus, participants could have adopted this narrow attentional zoom with the difficult cue task but spread attention more broadly with the easier cue task.

In Experiment 1, everything was the same except that the target and distractor locations were swapped, so that the distractor was now closer to the fixation than the target, and it was no longer easy to select a single region that included both target locations while excluding the distractor locations (see Fig. 3A). The cue task was still the same, and thus the perceptual load difference between the cue conditions should still be the same. Nonetheless, this change in stimulus configuration eliminated the effect of cue type: The interference from the distractor was just as strong regardless of the cueing condition.

The difference in the pattern of distractor interference between these two experiments cannot be attributed to PL, but it can be explained by differences in attentional zoom. The stimulus configuration in Experiment 2 makes it possible to narrow the attended region for the difficult cue task and still select the two possible target locations, which are relatively near the cue location at fixation. Participants can easily use narrow zoom in the difficult cue condition, which limits distractor interference, but they will use a wider zoom in the easy cue condition, leading to more interference. In Experiment 1, it is difficult to select the two possible target locations without also selecting the distractor locations. Participants use the same attentional zoom, regardless of the cue, and the interference is similar in both conditions. This pattern of interference is due to the ease in finding an effective attentional zoom setting, and not to PL.

Block order effects with go/no-go cues As described previously, some experiments have demonstrated that expectations can be set by an earlier block of trials, and the resulting effects of block order are difficult to reconcile with PLT. Order effects have been demonstrated in the go/no-go cue paradigm by Chen and Cave (2016), Experiment 4, suggesting that the size of the attentional zoom region is set differently depending on previous experience. Their participants saw stimulus displays that consisted of a target letter on the left or right of a centrally located go/no-go cue and a salient distractor letter above or below the center. The go/no-go cue required either an easy (color) or difficult (color + shape) discrimination. Each participant completed two blocks of trials: one with the easy cue task and one with the difficult cue task. The order of the blocks was counterbalanced across participants. The results depended on the order in which the two trial blocks were done. The participants who started with the easy cue showed distractor interference in the easy cue block but not in the difficult cue block. In contrast, the participants who started with the difficult cue showed no distractor interference in either the easy or the different cue block. Chen and Cave interpreted this block effect in the following way: if participants started with the easy cue, their attention was distributed more broadly, so that there was interference from the distractor. Once they moved on to the next block, the wide attentional zoom allowed too much interference for the difficult cue task, and thus the attended region was narrowed to limit the interference. If participants started with the difficult cue, they needed to have a narrow attentional zoom. That narrow zoom worked well for this task, and thus participants maintained a similar attentional setting into the easy cue condition so that distractor interference was low across the entire experiment for these participants.

Similar top-down effects have been found by Belopolsky, Zwaan, Theeuwes, and Kramer (2007), who investigated the effect of a color singleton on search efficiency as a function of top-down search strategy. Participants saw identical search displays in different blocks, but they were induced to adopt either a broad or a narrow attentional zoom in each block via a go/no-go response cue. The results show that a color singleton captured attention more in the broad attentional zoom condition than in the narrow one. Because the extent of attentional zoom and top-down search strategies are tightly linked, the results of these studies are in line with the notion that attentional zoom may have played an important role in the PL effects observed in previous studies.

\section{Interitem interference and the attentional changes to con-} trol it Chen and Cave's attentional zoom account is based on the idea that processing of a salient distractor depends not just on the amount of unused processing capacity or the number of nontargets in the display but also on the amount of interference among the different items in the display and the topdown adjustments in attentional allocation that are made to control this interference. Two other recent accounts are also centered around these factors. Both are based on considering the possible interactions among neural representations of the different stimulus items as they compete for representation.

A recently proposed account, the biased competition account of distractor processing (Scalf, Torralbo, Tapia, \& Beck, 2013; Torralbo \& Beck, 2008), offers one explanation of these interactions. According to the account, the degree of distractor processing is the result of a top-down biasing signal evoked by the need to resolve competitive interactions among neutral representations in visual cortex. The account assumes that all stimuli within the visual field compete for neural representation, and the competition can be biased by both bottom-up and top-down mechanisms (Desimone \& Duncan, 1995; Kastner, De Weerd, Desimone, \& Ungerleider, 1998; Reynolds, Chelazzi, \& Desimone, 1999). Furthermore, the degree of competitive interactions determines the amount of 
top-down bias needed to support the representation of the target, and this in turn affects the degree of exclusion of the distractor and the other stimuli in the display. Consequently, when the selection of the target is difficult, as in a typical high-load task, a strong top-down bias is needed to overcome the competitive interactions between the target and the nontargets, and this, in turn, results in the exclusion of the distractor and the other nontargets. In contrast, when the selection of the target is easy, as in a typical low-load or high-dilution condition, top-down bias is unnecessary due to the weak competitive interactions among the stimuli. This makes it unnecessary to have a narrow attentional window, resulting in distractor processing.

Because the amount of distractor interference is a function of the degree of competitive interactions among the stimuli in the visual field, the biased competition account is able to explain a variety of findings in the literature, including those found in (1) the PL studies that manipulate the number of nontargets or their homogeneity (e.g., Lavie, 1995; Lavie \& Cox, 1997); (2) the salience studies that manipulate the degradedness of the target and/or the distractor relative to each other and to the other stimuli in the display (e.g., Eltiti et al., 2005; Yeshurun \& Marciano, 2013); (3) the dilution studies that vary the similarity between the target and nontargets or the number of nontargets (e.g., Benoni \& Tsal, 2010; Tsal \& Benoni, 2010; Wilson et al., 2011). In addition, the biased competition account can explain why the degree of distractor interference should be smaller when the target and distractors or the target and nontargets are in the same hemisphere compared with when they are in different hemispheres (Torralbo \& Beck, 2008; Wei et al., 2013), and why there is no dilution when the nontargets are inverted letters or scrambled faces among upright letters or intact faces (Chen \& Cave, 2013; Thoma \& Lavie, 2013). Stimuli in the same hemisphere or category evoke stronger competitive interactions in visual cortex, which requires stronger top-down bias to support the target representation, resulting in the reduction or elimination of distractor processing.

Scalf et al. (2013) emphasize the top-down bias that favors the target over the other stimuli, but they describe this bias in very general terms. They do not describe this bias as an attentional zoom mechanism that distributes attention more narrowly as interference increases, although their evidence and their proposals seem generally consistent with the idea of attention zoom.

Another recent account gives a smaller role to top-down attentional adjustments to control attention. Neokleous et al. (2016) built a computer simulation of the interactions and interference among the different stimuli. Rather than attributing the results of PL and dilution experiments to changes in attentional control, they explain the results as differences in competition as interference levels vary. Their model includes mechanisms that have been part of many attentional theories over the years, including bottom-up attentional activations based on featural differences (salience) and competition among stimulus objects for representation. Like the earlier theory of visual attention (TVA; Giesbrecht et al., 2014; Kyllingsbæk et al., 2011), Neokleous et al.'s model is designed to explain the results of these experiments without any mechanism that explicitly implements the concept of perceptual load. The model has simulated the results from a small number of different experiments to show that this mechanism can provide accounts of results cited to support PLT as well as those used to support the dilution account.

Here we will mention two of those simulations. The model has not been applied to Lavie's experiments manipulating the number of nontargets, but it has been applied to Lavie and Cox's (1997) experiment in which a target letter (X or $\mathrm{N}$ ) appeared among similar distractor letters in the high-load condition and among multiple $O$ s in the low-load condition. The display contained a very salient distractor that was outside the search area, which produced higher target interference in the low-load condition than in the high-load condition. The model shows how Lavie and Cox's results might arise from bottom-up stimulus salience rather than from an attentional mechanism that allocates attention according to perceptual load.

To explore the model's response to dilution stimuli, Neokleous et al. (2016) simulated processing of the stimuli from Benoni and Tsal (2010), which includes low-load-lowdilution, low-load-high-dilution, and high-load-high-dilution conditions. In both high-dilution conditions, the addition of the extra nontargets diminishes activation of the distractor, leading to lower distractor interference, although the specific interactions among the different stimuli are different in each condition. Thus, the model produces results similar to Benoni and Tsal's experiment.

Neokleous et al. (2016) use these simulations to argue that a single model can account for the results from both perceptual load and dilution experiments, and that it can do so without mechanisms that are specially designed to produce perceptual load effects or dilution effects. Instead, these effects can arise from the bottom-up activation that objects receive when they differ from other objects in basic feature dimensions, because this activation can allow both target and nontargets to inhibit a salient distractor and can allow the target to overcome interference from that salient distractor.

The Neokleous et al. (2016) model, like the Scalf et al. (2013) account and the Chen and Cave (2013, 2014, 2016) attentional zoom account, portrays the different results across these different experiments as reflecting the different levels of interference among distractor items. In the Scalf et al. and the attentional zoom accounts, higher interference leads to more 
attentional control, which favors the target over other items in the display and thus limits the interference from a salient distractor. Neokleous et al. do not include a mechanism to limit the extent of attention in the face of difficulty; instead, the competition becomes more intense in their model, and the extra interference limits the ability of the distractor to influence processing.

The Neokleous (2016) model has the advantage of providing the most detailed account of attentional processing, but it cannot account for the results from Lavie's go-no-go experiments, in which the stimuli are identical across conditions, but distractor interference is reduced by a concurrent conjunction task. Those results can be explained if a conjunction task leads to narrower attentional zoom (Chen \& Cave, 2016) or to some other form of stronger top-down bias (Scalf et al., 2013). It may not be that difficult to augment Neokleous' model with an attentional zoom mechanism that varies according to the level of interference. The model already includes an "endogenous module" that provides top-down activation for items similar to a target representation, and this top-down mechanism might be expanded to limit nontarget processing when interference is high. This addition, however, would move the model away from its emphasis on late selection.

This model may also have difficulty accounting for Chen and Cave's (2013) finding that an upright letter target receives little interference from inverted letters, and Thoma and Lavie's (2013) finding that an intact face target receives little interference from scrambled faces. If new studies are able to confirm these results, it may be necessary to reconsider the level at which interference is generated in the model.

\section{Constraints imposed by the spatial nature of visual selec-} tion Underlying PLT is an assumption that selection of visual objects is extremely flexible and that objects can be easily included or excluded from attentional processing in order to make full use of the attentional resources. PLT assumes that all processing resources not needed to process the stimuli that are relevant to the current task will be allocated to other stimuli. The experiments summarized previously demonstrate that the link between relevance and selection is not always so strong and that a number of other factors play a role in determining the amount of attention allocated to distractors. Likewise, some of the results are not consistent with a simple dilution account; when the effects of abrupt onsets and blocked trials are eliminated, dilution only arises from stimuli within the attended region. Furthermore, accounts such as the model by Neokleous et al. (2016) provide alternative explanations of these results without invoking the concept of perceptual load.

There is another aspect of these results that is also not captured by either the PL or dilution accounts, although it has been included in other attentional theories. Experiments such at Chen and Cave's (2016) demonstrate that the allocation of spatial attention to nontargets depends to a large extent on the spatial arrangement of the stimuli. In Experiment 2 of this study, the two possible target locations are both within the same contiguous region, allowing distractors to be excluded more effectively than in Experiment 1, in which the possible distractor locations are between the possible target locations. The spatial constraints on attentional selection that are demonstrated in these experiments need to be included when describing the attentional interactions among objects in a complex scene. These spatial constraints have not been part of PLT, nor have they been explicitly included in explanations such as Eltiti et al.'s (2005) salience account, Gaspelin et al.'s (2014) slippage theory, the dilution accounts by Tsal and Benoni (2010) and Wilson et al. (2011), or the originally proposed version of the Neokleous et al. model.

Performance in tasks such as that in Chen and Cave (2016) suggests that in many cases, attention is allocated to a set of contiguous locations that together form a single region within the visual field. In fact, some have argued that attention is never split across noncontiguous regions (Jans, Peters, \& De Weerd, 2010). However, overall the evidence seems to suggest that splitting attention is possible but that it incurs a cost in mental effort (Cave, Bush, $\&$ Taylor, 2010), and thus the selected region might remain contiguous unless there is a compelling reason for it to be split. One conclusion to be drawn from these results is that when feasible, attentional selection will encompass a single region, and that region will have a fairly simple shape.

One specific type of spatial selection that was not included in either PLT or the Neokleous et al. model is the idea described earlier that the attended region can expand and contract to accommodate the needs of the current task, although this idea has been included in other attentional theories, including Eriksen and St. James's (1986) zoom lens metaphor, and the idea seems to be consistent with the proposals of Scalf et al. (2013). The possibility of selection by attentional zoom is illustrated in Fig. 4. Attentional zoom selects a region of the visual field, and thus the objects within this region can strongly activate their corresponding high-level representations while the objects outside this region are less able to. The size of the attentional zoom region is controlled by a mechanism that can be affected by many factors, including those factors that have been manipulated in previous PL experiments. In general, the attended region is narrowed when distractor interference makes it difficult to accurately find and identify the target.

Gauging interference As already noted, PLT did not specify precisely how perceptual load was defined, and Tsal and Benoni's (2010) dilution account was ambiguous about 


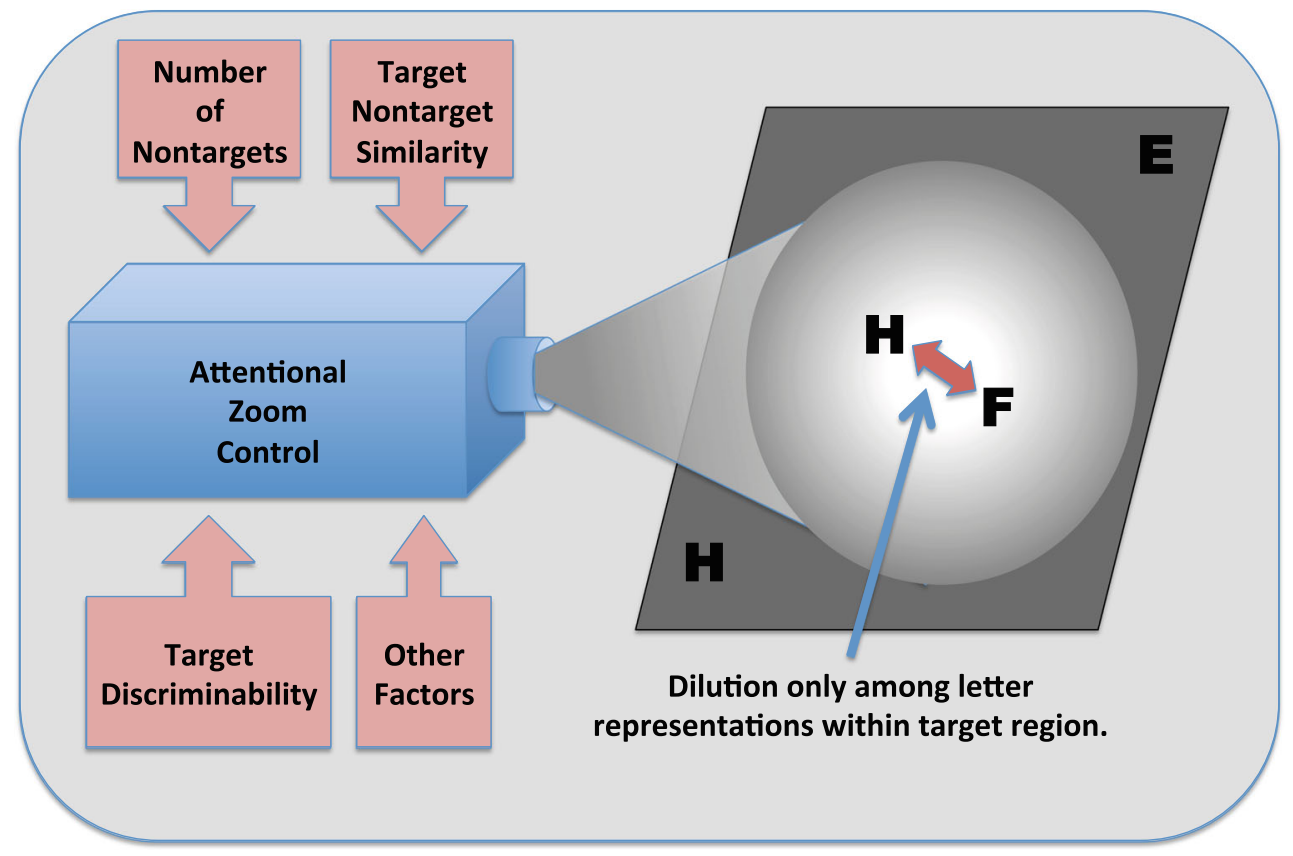

Fig. 4 A number of factors interact to determine the extent of attentional zoom. The red double-headed arrow represents dilution, which only occurs among representations of objects within the attentional zoom. (Color figure online)

exactly where in the stream of visual processing dilution is occurring. The attentional zoom account as suggested here also leaves some key aspects to be filled in later. It allows for the possibility that a number of different factors can affect how broadly attention can be distributed, but it leaves open the difficult question of how these different factors are combined to determine the appropriate attentional zoom setting. A full description of attentional zoom control will probably be fairly complex and may be best done with a computational model.

One possible component of that control mechanism, which was also suggested by Cave et al. (2010), is an "interference gauge" that can measure the degree to which target processing is being disrupted by distractor interference. By default, attention would be broadly distributed so that unexpected stimuli can be detected quickly. However, when the interference gauge detects high interference, the attended region zooms in more narrowly.

This arrangement recreates some aspects of PLT: When the number of nontargets is low or when they are not easily confused with targets, the wide attentional zoom setting allows more attention to be allocated to objects that are not relevant to the current task. Unlike PLT, however, this account acknowledges the important spatial constraints that limit how attention can be divvied up among multiple visual objects. It also acknowledges that the influence of factors such as target discriminability, target-nontarget similarity, and so forth, on attentional selection is indirect and subject to interactions with other factors.

Chen and Cave $(2013,2014,2016)$ saw the attentional zoom account as a way to explain Lavie's perceptual load experiments, and they concluded that attentional zoom was also an important factor in the dilution demonstrated by Benoni and Tsal (2010) and by Wilson et al. (2011). The factors that Lavie manipulated to increase perceptual load, such as adding more nontargets to the display or increasing the similarity between target and nontargets, might also lead to a narrowing of attentional zoom. The Neokleous et al. (2016) model accounts for some of these results without narrowing attentional zoom, but there are some shared assumptions underlying both systems: They both assume that these high-load manipulations cause additional competition among items. In the Neokleous model, this competition leads to more inhibition of the distractor, while in the attentional zoom account, it leads to narrower attentional zoom, which then limits processing of the distractor.

The results from Handy et al. (2001) and from Parks et al. (2013) show that models of attentional selection should include some mechanism for zooming and panning the attended region. It is probably fairly straightforward to add such a mechanism to the Neokleous model. With such an addition, the model may also be able to explain the results from the go/no-go perceptual load experiments that show reduced interference with a conjunction cue.

Conclusion As noted at the beginning, attentional selection allows some level of activation for multiple objects, and this simultaneous activation allows for complex interactions. The PLT and dilution accounts were attempts to identify general principles governing these interactions, but as experimental techniques are refined to produce more accurate measures of 
these interactions (Yantis \& Jonides, 1984, 1990), the emerging data show that the interactions are more complex than implied by these theories. Distractor interference has been used for many years in a variety of tasks to measure how fully distractors are processed, and the methods used to measure dilution by Tsal and Benoni (2010) and by Wilson et al. (2011) add to the existing paradigms in measuring the degree to which attention spreads to nontargets across the display. Future experiments using these methods are likely to lead to a better understanding of the interactions among target objects, nontarget objects, and selective attention.

Acknowledgments We thank Hermann Müller, Adam Biggs, and an anonymous reviewer for their helpful comments.

\section{References}

Awh, E., Belopolsky, A. V., \& Theeuwes, J. (2012). Top-down versus bottom-up attentional control: A failed theoretical dichotomy. Trends in Cognitive Sciences, 16, 437-443.

Bahrami, B., Carmel, D., Walsh, V., Rees, G., \& Lavie, N. (2008). Unconscious orientation processing depends on perceptual load. Journal of Vision, 8(12), 1-10.

Baylis, G. C., \& Driver, J. (1992). Visual parsing and response competition. Perception \& Psychophysics, 51, 145-162.

Belopolsky, A. V., Zwaan, L., Theeuwes, J., \& Kramer, A. F. (2007). The size of an attentional window modulates attentional capture by color singleton. Psychonomic Bulletin \& Review, 14, 934-938.

Benoni, H., \& Tsal, Y. (2010). Where have we gone wrong? Perceptual load does not affect selective attention. Vision Research, 50, 1292-1298.

Benoni, H., \& Tsal, Y. (2012). Controlling for dilution while manipulating load: Perceptual and sensory limitations are just two aspects of task difficulty. Psychonomic Bulletin \& Review, 19, 631-638.

Benoni, H., \& Tsal, Y. (2013). Conceptual and methodological concerns in the theory of perceptual load. Frontiers in Psychology, 4(522), 1-7.

Benoni, H., Zivony, A., \& Tsal, Y. (2014). Attentional sets influence perceptual load effects, but not dilution effects. The Quarterly Journal of Experimental Psychology, 67, 785-792.

Biggs, A. T., \& Gibson, B. S. (2010). Competition between color salience and perceptual load during visual selection can be biased by topdown set. Attention, Perception \& Performance, 72, 53-64.

Biggs, A. T., \& Gibson, B. S. (2013). Learning to ignore salient color distractors during serial search: Evidence for experience-dependent attention allocation strategies. Frontiers in Psychology, 4(326), 1-13.

Biggs, A. T., \& Gibson, B. S. (2014). Visual salience can co-exist with dilution during visual selection. Journal of Experimental Psychology: Human Perception and Performance, 41, 7-14.

Biggs, A. T., Kreager, R. D., \& Davoli, C. C. (2015). Finding a link between guided search and perceptual load theory. Journal of Cognitive Psychology, 27, 164-179.

Biggs, A. T., Kreager, R. D., Gibson, B. S., Villano, M., \& Crowell, C. R. (2012). Semantic and affective salience: The role of meaning and preference in attentional capture and disengagement. Journal of Experimental Psychology: Human Perception and Performance, 38, 531-541.

Broadbent, A. D., Cooper, P. E., FitzGerald, P., \& Parks, K. R. (1982). Cognitive Failures Questionnaire (CFQ) and its correlates. British Journal of Clinical Psychology, 21, 1-16.
Brand-D'Abrescia, M., \& Lavie, N. (2007). Distractor effects during processing of words under load. Psychonomic Bulletin \& Review, 14, 1153-1157.

Carmel, D., Saker, P., Rees, G., \& Lavie, N. (2007). Perceptual load modulates conscious flicker perception. Journal of Vision, 7(14), $1-13$.

Cartwright-Finch, U., \& Lavie, N. (2007). The role of perceptual load in inattentional blindness. Cognition, 102, 321-340.

Cave, K. R., Bush, W. S., \& Taylor, T. G. G. (2010). Split attention as part of a flexible attentional system for complex scenes: Comment on Jans, Peters, and De Weerd (2010). Psychological Review, 117, 685-696.

Chen, Z. (2003). Attentional focus, processing load, and Stroop interference. Perception \& Psychophysics, 65, 888-900.

Chen, Z., \& Cave, K. R. (2013). Perceptual load vs. dilution: The role of attentional focus, stimulus category, and target predictability. Frontiers in Psychology, 4(327), 1-14.

Chen, Z., \& Cave, K. R. (2014). Constraints on dilution from a narrow attentional zoom reveal how spatial and color cues direct selection. Vision Research, 101, 125-137.

Chen, Z., \& Cave, K. R. (2015). Singleton search is guided by knowledge of the target, but maybe it shouldn't be. Vision Research, 115, 92-103.

Chen, Z., \& Cave, K. R. (2016). Zooming in on the cause of the perceptual load effect in the go/nogo paradigm. Journal of Experimental Psychology: Human Perception and Performance. doi:10.1037/ xhp0000168

Chen, Z., \& Chan, C. C. (2007). Distractor interference stays constant despite variation in working memory load. Psychonomic Bulletin \& Review, 14, 306-312.

Cosman, J. D., \& Vecera, S. P. (2009). Perceptual load modulates attentional capture by abrupt onsets. Psychonomic Bulletin \& Review, 16, 404-410

Cosman, J. D., \& Vecera, S. P. (2010). Attentional capture under high perceptual load. Psychonomic Bulletin \& Review, 17, 815-820.

Cosman, J. D., \& Vecera, S. P. (2012). Object-based attention overrides perceptual load to modulate visual distraction. Journal of Experimental Psychology: Human Perception and Performance, $38,576-579$.

Couperus, J. W. (2001). Perceptual load influences selective attention across development. Developmental Psychology, 47, 1431-1439.

de Fockert, J. W. (2013). Beyond perceptual load and dilution: A review of the role of working memory in selective attention. Frontiers in Psychology, 4(287), 1-12.

Desimone, R., \& Duncan, J. (1995). Neural mechanisms of selective visual attention. Annual Review of Neuroscience, 18, 193-222.

Eltiti, S., Wallace, D., \& Fox, E. (2005). Selective target processing: Perceptual load or distractor salience? Perception \& Psychophysics, 67, 876-885.

Eriksen, B. A., \& Eriksen, C. W. (1974). Effects of noise letters upon the identification of a target letter in a nonsearch task. Perception \& Psychophysics, 16, 143-149.

Eriksen, C. W., \& Hoffman, J. E. (1973). The extent of processing of noise elements during selective encoding from visual display. Perception \& Psychophysics, 14, 155-160.

Eriksen, C. W., \& St. James, J. D. (1986). Visual attention within and around the field of focal attention: A zoom lens model. Perception \& Psychophysics, 40, 225-240.

Forster, S. (2013). Distraction and mind-wandering under load. Frontiers in Psychology, 4(283), 1-6.

Forster, S., \& Lavie, N. (2007). High perceptual load makes everybody equal. Psychological Science, 18, 377-381.

Forster, S., \& Lavie, N. (2008a). Attentional capture by entirely irrelevant distractors. Visual Cognition, 16, 200-214.

Forster, S., \& Lavie, N. (2008b). Failures to ignore entirely irrelevant distractors: The role of load. Journal of Experimental Psychology: Applied, 14, 73-83. 
Forster, S., \& Lavie, N. (2009). Harnessing the wandering mind: The role of perceptual load. Cognition, 111, 345-355.

Gaspelin, N., Ruthruff, E., \& Jung, K. (2014). Slippage theory and the flanker paradigm: An early-selection account of selective attention failures. Journal of Experimental Psychology: Human Perception and Performance, 40, 1257-1273.

Gaspelin, N., Ruthruff, E., Jung, K., Cosman, J. D., \& Vecera, S. P. (2012). Does low perceptual load enable capture by colour singletons? Journal of Cognitive Psychology, 24, 735-750.

Giesbrecht, B., Sy, J., Bundesen, C., \& Kyllingsbæk, S. (2014). A new perspective on the perceptual selectivity of attention under load. Annals of the New York Academy of Sciences, 1316, 71-86.

Handy, T. C., \& Mangun, G. R. (2000). Attention and spatial selection: Electrophysiological evidence for modulation by perceptual load. Perception \& Psychophysics, 62, 175-186.

Handy, T. C., Soltani, M., \& Mangun, G. R. (2001). Perceptual load and visuocortical processing: Event-related potentials reveal sensorylevel selection. Psychological Science, 12, 213-217.

Hoffman, J. E. (1979). A two-stage model of visual search. Perception \& Psychophysics, 25, 319-327.

Huang-Pollock, C. L., Carr, T. H., \& Nigg, J. T. (2002). Development of selective attention: Perceptual load influences early versus late selection in children and adults. Developmental Psychology, 38, 363-375.

Jans, B., Peters, J. C., \& De Weerd, P. (2010). Visual spatial attention to multiple locations at once: The jury is still out. Psychological Review, 117, 637-684.

Johnson, D. N., McGrath, A., \& McNeil, C. (2002). Cuing interacts with perceptual load in visual search. Psychological Science, 13, 284-287.

Kahneman, D., \& Chajczyk, D. (1983). Tests of the automaticity of reading: Dilution of Stroop effects by color-irrelevant stimuli. Journal of Experimental Psychology: Human Perception and Performance, 9, 497-509.

Kastner, S., De Weerd, P., Desimone, R., \& Ungerleider, L. G. (1998). Mechanisms of directed attention in the human extrastriate cortex as revealed by functional MRI. Science, 282, 108-111.

Kumada, T., \& Humphreys, G. W. (2002). Early selection induced by perceptual load in a patient with frontal lobe damage: External vs. internal modulation of processing control. Cognitive Neuropsychology, 19, 49-65.

Kyllingsbæk, S., Sy, J. L., \& Giesbrecht, B. (2011). Understanding the allocation of attention when faced with varying perceptual load in partial report: A computational approach. Neuropsychologia, 40, $1487-1497$

Lavie, N. (1995). Perceptual load as a necessary condition for selective attention. Journal of Experimental Psychology: Human Perception and Performance, 21, 451-468.

Lavie, N. (2005). Distracted and confused? Selective attention under load. Trends in Cognitive Sciences, 9, 75-82.

Lavie, N., \& Cox, S. (1997). On the efficiency of visual selective attention: Efficient visual search leads to inefficient distractor rejection. Psychological Science, 8, 395-398.

Lavie, N., \& Tsal, Y. (1994). Perceptual load as a major determinant of the locus of selection in visual attention. Perception \& Psychophysics, 56, 183-197.

Leber, A. B., \& Egeth, H. E. (2006a). Attention on autopilot: Past experience and attentional set. Visual Cognition, 14, 565-583.

Leber, A. B., \& Egeth, H. E. (2006b). it's under control: Top-down search strategies can override attentional capture. Psychonomic Bulletin \& Review, 13, 132-138.

Martin, M. (1983). Cognitive failure: Everyday and laboratory performance. Bulletin of the Psychonomic Society, 21, 97-100.

Mevorach, C., Tsal, Y., \& Humphreys, G. (2014). Low level perceptual, not attentional, processes modulate distractor interference in high perceptual load displays: Evidence form neglect/extinction. Frontiers in Psychology, 4(966), 1-8.

Miller, J. (1991). The flanker compatibility effect as a function of visual angle, attentional focus, visual transients, and perceptual load: A search for boundary condition. Perception \& Psychophysics, 49, 270-288.

Muggleton, N., Lamb, R., Walsh, V., \& Lavie, N. (2008). Perceptual load modulates visual cortex excitability to magnetic stimulation. Journal of Neurophysiology, 100, 516-519.

Murphy, G., Groeger, J. A., \& Greene, C. M. (2016). Twenty years of load theory -Where are we now, and where should we go next? Psychonomic Bulletin \& Review. doi:10.3758/s13423-015-0982-5

Murray, J. E., \& Jones, C. (2002). Attention to local form information can prevent access to semantic information. The Quarterly Journal of Experimental Psychology A, 55, 609-625.

Neisser, U. (1967). Cognitive psychology. Englewood Cliffs, NJ: Prentice Hall.

Neokleous, K., Shimi, A., \& Avraamides, M. N. (2016). Modeling the effects of perceptual load: Saliency, competitive interactions, and top-down biases. Frontiers in Psychology, 7, 1-15.

Paquet, L., \& Craig, G. L. (1997). Evidence for selective target processing with a low perceptual load flankers task. Memory \& Cognition, 25, 182-189.

Paquet, L., \& Lortie, C. (1990). Evidence for early selection: Precuing target location reduces interference from same category distractors. Perception \& Psychophysics, 48, 382-388.

Parks, N. A., Beck, D. M., \& Kramer, A. F. (2013). Enhancement and suppression in the visual field under perceptual load. Frontiers in Psychology, 4(275), 1-8.

Reynolds, J. H., Chelazzi, L., \& Desimone, R. (1999). Competitive mechanisms subserve attention in macaque areas V2 and V4. Journal of Neuroscience, 19, 1736-1753.

Ro, T., Friggel, A., \& Lavie, N. (2009). Musical expertise modulates the effects of visual perceptual load. Attention, Perception, \& Psychophysics, 71, 671-674.

Roper, Z. J., Cosman, J. D., Mordkoff, T., \& Vecera, S. P. (2011). Perceptual load effect is determined by resource demand and data limitation. Journal of Vision, 11, 247.

Roper, Z. J., Cosman, J. D., \& Vecera, S. P. (2013). Perceptual load corresponds with factors known to influence visual search. Journal of Experimental Psychology: Human Perception and Performance, 39, 1340-1351.

Roper, Z. J., \& Vecera, S. P. (2013). Response terminated displays unload selective attention. Frontiers in Psychology, 4(967), 1-10.

Scalf, P. E., Torralbo, A., Tapia, E., \& Beck, D. M. (2013). Competition explains limited attention and perceptual resources: Implications for perceptual load and dilution theories. Frontiers in Psychology, $4(243), 1-9$.

Serences, J. T., \& Yantis, S. (2006). Selective visual attention and perceptual coherence. Trends in Cognitive Sciences, 10, 38-45.

Taya, S., Adams, W. J., Graf, E. W., \& Lavie, N. (2009). The fate of taskirrelevant visual motion: Perceptual load versus feature-based attention. Journal of Vision, 9(12), 1-10.

Theeuwes, J., Kramer, A. F., \& Belopolsky, A. V. (2004). Attentional set interacts with perceptual load in visual search. Psychonomic Bulletin \& Review, 11, 697-702.

Thoma, V., \& Lavie, N. (2013). Perceptual load effects on processing distractor faces indicate face-specific capacity limits. Visual Cognition, 21, 1053-1076.

Torralbo, A., \& Beck, D. M. (2008). Perceptual-load-induced selection as a result of local competitive interactions in visual cortex. Psychological Science, 19, 1045-1050.

Treisman, A., \& Gelade, G. (1980). A feature integration theory of attention. Cognitive Psychology, 12, 97-136. 
Tsal, Y., \& Benoni, H. (2010). Diluting the burden of load: Perceptual load effects are simply dilution effects. Journal of Experimental Psychology: Human Perception and Performance, 36, 1645-1656.

Wei, P., Kang, G., \& Zhou, X. (2013). Attentional selection within and across hemispheres: Implications for the perceptual load theory. Experimental Brain Research, 225, 37-45.

Wilson, D. E., Muroi, M., \& MacLeod, C. M. (2011). Dilution, not load, affects distractor processing. Journal of Experimental Psychology: Human Perception and Performance, 37, 319-335.

Yantis, S. (2000). Goal-directed and stimulus-driven determinants of attentional control. In S. Monsell \& J. Driver (Eds.), Attention and performance, XVIII (pp. 73-103). Cambridge, MA: MIT Press.

Yantis, S. (2008). The neural basis of selective attention: Cortical sources and targets of attentional modulation. Current Directions in Psychological Science, 17, 86-90.

Yantis, S., \& Jonides, J. (1984). Abrupt visual onsets and selective attention: evidence from visual search. Journal of Experimental Psychology: Human Perception and Performance, 10, 601-621.
Yantis, J., \& Jonides, J. (1990). Abrupt visual onsets and selective attention: voluntary vs. automatic allocation. Journal of Experimental Psychology: Human Perception and Performance, 16, 121-134.

Yeh, Y.-Y., Lee, S.-M., Chen, Y.-H., \& Chen, Z. (2014). Selection history modulates the effects of dual mechanisms on flanker interference. Journal of Experimental Psychology: Human Perception and Performance, 40, 2038-2055.

Yeh, Y.-Y., \& Lin, S.-H. (2013). Two mechanisms of distractor dilution: Visual selection in a continuous flow. Journal of Experimental Psychology: Human Perception and Performance, 39, 872-892.

Yeshurun, Y., \& Marciano, H. (2013). Degraded stimulus visibility and the effects of perceptual load on distractor interference. Frontiers in Psychology, 4(289). doi:10.3389/fpsyg.2013.00289

Zehetleitner, M., Goschy, H., \& Müller, H. J. (2012). Top-down control of attention: It's gradual, practice dependent, and hierarchically organized. Journal of Experimental Psychology: Human Perception and Performance, 38, 941-957. 